\title{
Overview of Structural Reliability Analysis Methods - Part I: Local Reliability Methods
}

\author{
ChangWu HUANG ${ }^{1,{ }^{*}}$, Abdelkhalak El Hami ${ }^{1}$, Bouchaïb Radi ${ }^{2}$ \\ ${ }^{1}$ Normandie Université, INSA Rouen, LOFIMS, 76000 Rouen, France. \\ * Corresponding author: ChangWu.Huang.PRC@gmail.com \\ ${ }^{2}$ LIMII, FST Settat, BP: 577, Route de Casa, Settat, Morocco.
}

\begin{abstract}
Our work presents an overview of structural reliability analysis, which plays important roles in structural design. In Part I of the overview, the so-called local reliability methods are summarized. The term "local reliability methods" refers to reliability analysis methods that use the local approximate of actual limit state function in calculation of failure probability. From this perspective of view, the mean value first-order second moment (MVFOSM) method, design pointbased methods (FORM/SORM and RSM) are included in local reliability methods. Local reliability methods are basic approaches for reliability analysis and commonly used in research and applications.

KEYWORDS. Reliability Analysis, Limit State Function, Design Point, Local Reliability Methods.
\end{abstract}

\section{Introduction}

Structural design, in general, aims at achieving structures that satisfy safety criteria, serviceability and durability under specified service conditions. Since uncertainty is ubiquitous in engineering design, incorporation of uncertainties in engineering design is essentially required[1]. Reliability analysis offers the theoretical framework for considering uncertainties in engineering decision scheme[2]0. Reliability can be defined as the probability that a structure or system can perform a required function under specified service conditions during a given period of time [3]0. Conversely, the failure probability (or probability of failure) is the probability that a structure does not perform satisfactorily within a given period of time and stated conditions.

As many different reliability analysis methods exist, these methods are firstly grouped into some categories. Inspired by the classification in Reference [4]0, the existed reliability analysis methods are grouped into three main categories: Local Reliability Methods, Sampling Methods and Global Reliability Methods.

This paper, the Part I of the overview of structural reliability analysis methods, focus on the first category reliability methods, i.e., local reliability methods. The following part of the paper is organized as follows. Some basics for reliability analysis is firstly presented in Section 2. Then local reliability methods are detailed in Section 40. An example is illustrated in Section 0. Finally, a brief summary is given in Section 5.

\section{Basic Concepts for Reliability Analysis}

In structural reliability problems, the input uncertain variables that governs the problem are modeled as basic random variables, and the space of basic random variables is divided into the failure and safe regions by the so-called limit state function (or surface). Thus, the probability of failure is calculated by integrating the joint probability density of the random variables over the failure region.

\subsection{Performance and Limit State Function}

In reliability analysis, the so-called limit state plays an important role. A limit state is a specific condition of a structure beyond which the structure no longer fulfills the relevant criterion on its function [5]. In order to quantitatively analysis the reliability of a structure, the response and limit states of the structure should be interpreted and formulated by a mathematical model. The basic random 
variables $\mathbf{X}=\left[X_{1}, X_{2}, \cdots, X_{n}\right]^{\mathrm{T}}$, which represent the input uncertain variables that govern the performance (response) of the structure, are used to construct a mathematical formulation to present the performance of the structure [6][7]. Consequently, the performance function, which describes the performance of a structure, can be defined as a function of the basic random variables

$$
Z=G(\mathbf{X})=G\left(X_{1}, X_{2}, \cdots, X_{n}\right)
$$

in such a way that $Z=G(\mathbf{X})>0$ indicates the structure is in safe state, i.e., the structure can function normally, and $Z=G(\mathbf{X})<0$ implies the state of failure, i.e., the structure cannot perform as required in this situation. The boundary $Z=G(\boldsymbol{X})=0$ between the safe domain $\mathcal{S}=\{\mathbf{x} \mid G(\mathbf{x})>0\}$ and the failure domain $\mathcal{F}=\{\mathbf{x} \mid G(\mathbf{x}) \leq 0\}$ is the so-called limit-state function [8], as shown in Figure 1. The concrete form of the performance function is usually obtained by structural analysis.

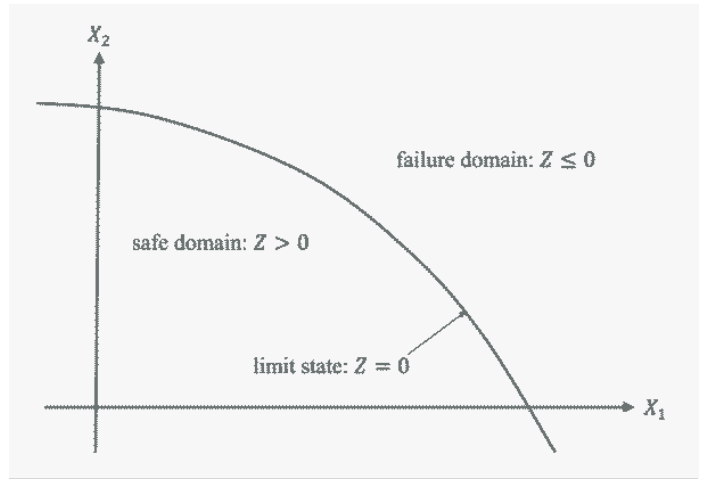

Figure 1. Limit state function and failure and safe domains

In practical reliability problems, the basic random variables may be statistically dependent and nonnormally distributed. This indeed brings complexities and makes the nontrivial reliability problem more complicate. In order to simplify the computation, the basic random variables are generally transformed into independent standard normal variables [9]. Probability transformation constructs a transformation $T$ that converts basic random vector $\mathbf{X}$ to a standard normal random vector $\mathbf{U}=\left[U_{1}, \cdots, U_{n}\right]^{\mathrm{T}}$, and correspondingly transforms the performance function from $\mathbf{X}$-space to standard normal space (U-space), i.e.,

$$
\mathbf{u}=T(\mathbf{x}) \quad \text { and } \quad H(\mathbf{u})=G\left(T^{-1}(\mathbf{x})\right)
$$

where $\mathbf{u}$ and $\mathbf{x}$ are the realizations of $\mathbf{x}$ and $\mathbf{U}$, respectively, $H(\mathbf{u})$ is the corresponding performance function in standard normal space (U-space). Then, the reliability analysis problem can be solved in $\mathbf{U}$-space, which is easier than in $\mathbf{X}$-space. The transformation can be performed in several ways according to the available statistical information and properties of the basic random variables. Details for probability transformation can be found in [9][10] [11].

\subsection{Failure Probability and Reliability Index}

Since structural reliability is defined as the probability that a structure can perform as required under specified service conditions during a given period of time, it can be measured by the probability of the structure in safe domain $p_{\mathrm{s}}$. The complementary probability is the failure probability $p_{\mathrm{f}}$, which signifies the probability that the structure cannot function normally. Obviously, the state of structure is safe or otherwise failure, and thus it has $p_{\mathrm{s}}+p_{\mathrm{f}}=1$. Therefore, either $p_{\mathrm{s}}$ or $p_{\mathrm{f}}$ can be used to represent the reliability of a structure. Commonly, the failure probability (or probability of failure) is used to signify the reliability level of a structure. 
With above definitions, failure probability is determined by integration over the failure domain:

$$
\begin{aligned}
p_{\mathrm{f}} & =\mathrm{P}(G(\mathbf{X}) \leq 0)=\int_{G(\mathbf{X}) \leq 0} f_{X}(\mathbf{x}) \mathrm{d} \mathbf{x} \\
& =\mathrm{P}(H(\mathbf{U}) \leq 0)=\int_{H(\mathbf{U}) \leq 0} \phi_{U}(\mathbf{u}) \mathrm{d} \mathbf{u}
\end{aligned}
$$

where $\mathrm{P}(\cdot)$ is the probability operator, $f_{X}(\mathbf{x})$ is the joint probability density function (PDF) of random vector $\mathbf{X}, \phi_{U}(\mathbf{u})$ is the standard multidimensional Gaussian density function. In Equation (3), the first line calculates the failure probability in $\mathbf{X}$-space, whereas the second line performs the calculation in $\mathbf{U}$-space. Thus, the main task of reliability analysis problem is to estimate the failure probability defined in Equation (3). This problem is challenging because, in practice, the joint probability density function $f_{X}(\mathbf{x})$ is hard to obtain, and, even if this function is available, the evaluation of the multiple integral is complicate and difficult.

\subsubsection{Cornell Index}

As directly using the integration in Equation (3) to calculate the failure probability is impracticable, it is convenient to use the reliability index $\beta$, which has a corresponding relation with failure probability, to express the reliability. It is obvious that the failure probability depends on the distribution of performance function. For a simple $R-S$ case with performance function $Z=G(R, S)=R-S$, the resistance $R$ and loads effect $S$ are assumed to be normally distributed and uncorrelated. Thus, the structure's performance $z$ is also normally distributed, i.e., $Z \sim \mathcal{N}\left(\mu_{z}, \sigma_{z}^{2}\right)$ where $\mu_{z}$ and $\sigma_{z}$ are the mean value and standard deviation of the performance, and the PDF of $z$ in this case is

$$
f_{Z}(z)=\frac{1}{\sqrt{2 \pi} \sigma_{Z}} \exp \left[-\frac{1}{2}\left(\frac{z-\mu_{Z}}{\sigma_{Z}}\right)^{2}\right]
$$

Accordingly, the failure probability can be computed as

$$
\begin{aligned}
p_{f} & =\mathrm{P}(Z \leq 0)=\int_{-\infty}^{0} f_{Z}(z) \mathrm{d} z \\
& =\int_{-\infty}^{0} \frac{1}{\sqrt{2 \pi} \sigma_{Z}} \exp \left[-\frac{1}{2}\left(\frac{z-\mu_{Z}}{\sigma_{Z}}\right)^{2}\right] \mathrm{d} z \\
& =\Phi\left(-\frac{\mu_{Z}}{\sigma_{Z}}\right)=\Phi\left(-\beta_{\mathrm{C}}\right)
\end{aligned}
$$

where $\Phi(\cdot)$ is the CDF of standard normal distribution, and $\beta_{\mathrm{C}}=\mu_{z} / \sigma_{z}$ is the so-called Cornell reliability index, named after Cornell [12]. The Cornell reliability index is the first and simplest reliability index, which was used as early as 1969. This reliability index can be seen as accurate only when the performance function is normally distributed, otherwise it may give inadequate results. Furthermore, the Cornell reliability index is lack of invariance for equivalent performance functions [13].

\subsubsection{Hasofer-Lind Index}

The Hasofer-Lind reliability index $\beta_{\text {нь }}$ proposed by Hasofer and Lind in 1974 overcomes the disadvantages of Cornell reliability index. The Hasofer-Lind reliability index $\beta_{\text {нц }}$ is defined as the shortest Euclidean distance from the origin to the limit state surface in the standard normal space [14]. Thus, $\beta_{\mathrm{HL}}$ is obtained by solving the optimization problem

$$
\beta_{\mathrm{HL}}=\min \|\mathbf{u}\|=\left(\mathbf{u}^{\mathrm{T}} \mathbf{u}\right)^{1 / 2} \quad \text { s.t. } H(\mathbf{u})=0
$$

where $\mathbf{u}$ and $H(\mathbf{u})=G\left(T^{-1}(\mathbf{u})\right)$ are transformed standard normal random vector and corresponding performance function, which have been described in Equation (2). The reliability index $\beta_{\mathrm{HL}}$ is related to the design point, which is of importance for FORM and SORM and is detailed below. 


\section{Local Reliability Methods}

The mean value first-order second moment (MVFOSM) method, first-order reliability methods (FORM), second-order reliability method (SORM) and response surface method (RSM) are classified in Local Reliability Methods in this paper. The MVFOSM estimates the mean and variance of the response by using first and second moments of the random variables. In FORM, the actual limit state function is approximated by its first-order Taylor expansion at the design point (also named as the most probable point or MPP). SORM approximates the limit state function by second-order Taylor expansion at the design point. While, RSM usually uses a second-order polynomial to substitute the implicit limit state function around the design point. It is obvious that these methods, MVFOSM, FORM, SORM and RSM, all use local approximation of the actual limit state function in calculation of the failure probability. This is the implication of the term 'local reliability methods'.

\subsection{Mean Value First-Order Second Moment (MVFOSM) Method}

The mean value first-order second moment (MVFOSM) method, or simplified as mean value (MV) method, is the simplest and least expensive reliability method. This method estimates the mean and variance of the performance function based on the first-order Taylor series approximation of the performance function and its derivatives at the mean values of the random input variables. Then these two statistics (mean and variance) of the performance function are used to calculate the reliability index.

In MVFOSM method, the mean and variance of the performance function $\mu_{G}$ and $\sigma_{G}^{2}$, reliability index $\beta$, and the probability of failure $p_{\mathrm{f}}$ are computed by

$$
\begin{aligned}
& \mu_{G}=G\left(\mu_{X}\right) \\
& \sigma_{G}^{2}=\sum_{i=1}^{n} \sum_{j=1}^{n} \frac{\partial G\left(\mu_{X}\right)}{\partial X_{i}} \frac{\partial G\left(\mu_{X}\right)}{\partial X_{j}} \operatorname{Cov}\left(X_{i}, X_{j}\right) \\
& \beta=\frac{\mu_{G}}{\sigma_{G}} \\
& p_{\mathrm{f}}=\Phi(-\beta)
\end{aligned}
$$

where $\mu_{X}$ represents the mean of the input random vector $\mathbf{X}, \operatorname{Cov}\left(X_{i}, X_{j}\right)$ is the covariance of $X_{i}$ and $X_{j}$, and $\Phi$ is the standard Normal cumulative distribution function (CDF).

Above approximations of the mean and variance of performance function are sufficiently accurate only when the performance function is nearly linear and the input random variables are approximately Gaussian. In other situations, MVFOSM method does not guarantee the accuracy. Another drawback of MVFOSM method is that it fails to be invariant with different mathematically equivalent formulations of the same problem.

\subsection{Design Point-Based Methods}

The design point-based methods attempted to overcome the limitations of MFOSM method by approximating the limit state function at the optimal point, called design point, in uncorrelated standard normal space. This kind of method contains two approaches: the first-order and second-order reliability methods (FORM and SORM).

\subsubsection{Design Point}

The design point search is performed in uncorrelated standard normal space ("U-space") rather than in the origin actual variables space ("X-space"), which may be correlated and non-normal. The transformation from correlated non-normal variables (in $\mathbf{X}$-space) to uncorrelated standard normal variables 
(in $\mathbf{U}$-space) is denoted as $\mathbf{U}=T(\mathbf{X})$ and its inverse transformation is noted by $\mathbf{X}=T^{-1}(\mathbf{U})$. With the transformation of random variables, the failure probability is computed in $\mathbf{U}$-space as

$$
p_{\mathrm{f}}=\int_{G(X) \leq 0} f_{X}(\mathbf{x}) \mathrm{d} \mathbf{x}=\int_{H(U) \leq 0} \phi_{U}(\mathbf{u}) \mathrm{d} \mathbf{u}
$$

where $H(\mathbf{U})=G\left(T^{-1}(\mathbf{X})\right)$ is the performance function in $\mathbf{U}$-space, $\phi_{U}(\mathbf{u})$ is the joint PDF of $\mathbf{U}$.

The design point $\mathbf{u}^{*}$ is defined by the constrained optimization problem

$$
\mathbf{u}^{*}=\arg \min \{\|\mathbf{u}\| \mid H(\mathbf{u}) \leq 0\}
$$

where "argmin" denotes the argument of the minimum of a function. It can be seen that the design point $\mathbf{u}^{*}$ is in failure domain, $H(\mathbf{u}) \leq 0$, and has the minimum distance from the origin in the standard normal space. On account of that the equal probability density contours in the standard normal space are concentric circles centered at the origin, the point $u^{*}$ has the highest probability density among all realizations in the failure domain $H(\mathbf{u}) \leq 0$. That is the reason that, in the reliability community, the point $\mathbf{u}^{*}$ is commonly called the design point, or other names such as most probable point (MPP) and $\beta$-point.

It can be seen from the definition of the $\mathbf{u}^{*}$ in $\mathbf{U}$-space that the neighborhood of this point makes a dominant contribution to the last integral in Equation (11). In this sense, the design point u is an optimal point for the linearization of the limit state function. Another important advantage of the design point is that it is invariant of the formulation of the limit state function, even though it may be dependent on the selected form of the transformation from $\mathbf{x}$ to $\mathbf{U}$ space.

Since the design point is of paramount importance, several iterative algorithms are proposed to solve Equation (12), in order to obtain $\mathbf{u}^{*}$. The widely used HL-RF algorithm, which as firstly proposed by Hasofer and Lind [14] and later generalized for non-normal variables by Rackwitz and Fissler [15], is presented here. Starting from an initial point $\mathbf{u}^{(0)}=T\left(\mathbf{x}^{(0)}\right)$, the iterative formula of HL-RF algorithm is

$$
\mathbf{u}^{(i+1)}=\left[H\left(\mathbf{u}^{(i)}\right) /\left\|\nabla H\left(\mathbf{u}^{(i)}\right)\right\|+\alpha^{(i)} \mathbf{u}^{(i)}\right]\left(\alpha^{(i)}\right)^{\mathrm{T}}
$$

where the superscript $(i)$ signifies the $i$-th iteration, $\nabla H\left(\mathbf{u}^{(i)}\right)=\left[\partial H\left(\mathbf{u}^{(i)}\right) / \partial U_{1}, \cdots, \partial H\left(\mathbf{u}^{(i)}\right) / \partial U_{n}\right]$ is the gradient row vector at $\mathbf{u}^{(i)}$, and $\alpha^{(i)}=-\nabla H\left(\mathbf{u}^{(i)}\right) /\left\|\nabla H\left(\mathbf{u}^{(i)}\right)\right\|$ is the normalized negative gradient row vector. For most problems, this algorithm works well and converges in just a few steps. In some special situation, the HL-RF algorithm may fail in convergence. Furthermore, in order to obtain the global solution of the problem, it is good practice to repeat the solution starting from a different initial point $\mathbf{x}^{(0)}$.

\subsubsection{First-Order Reliability Method (FORM)}

In first-order reliability method (FORM), the limit state function in $\mathbf{U}$-space, $H(\mathbf{u})=0$, is linearized by the first-order Taylor expansion at the design point $\mathbf{u}^{*}$. Considering $H\left(\mathbf{u}^{*}\right)=0$, the linearized limit state function is written as

$$
H(\mathbf{u}) \cong H_{1}(\mathbf{u})=\nabla H\left(\mathbf{u}^{*}\right)\left(\mathbf{u}-\mathbf{u}^{*}\right)=\left\|\nabla H\left(\mathbf{u}^{*}\right)\right\|(\beta-\alpha \mathbf{u})
$$

where $H_{1}(\mathbf{u})$ denotes the first-order approximation of $H(\mathbf{u}), \nabla H(\mathbf{u})=\left[\partial H(\mathbf{u}) / \partial U_{1}, \cdots, \partial H(\mathbf{u}) / \partial U_{n}\right]$ is the gradient row vector, $\alpha=-\nabla H\left(\mathbf{u}^{*}\right) /\left\|\nabla H\left(\mathbf{u}^{*}\right)\right\|$ is the normalized negative gradient row vector at the design point (an unit vector normal to the limit state surface at the design point and pointing towards the failure domain), and $\beta=\alpha \mathbf{u}^{*}$ is the reliability index. 
From above, the limit state surface $H(\mathbf{u})=0$ is linearized by $\beta-\alpha \mathbf{u}=0$. Correspondingly, the failure domain $H(\mathbf{u}) \leq 0$ is replaced by the half space $\beta-\alpha \mathbf{u} \leq 0$, as shown in Figure 2. Thus, the first-order approximation of the failure probability is given by the probability content of the half space in the standard normal space [9], that is,

$$
p_{\mathrm{f}} \cong p_{\mathrm{f} 1}=\Phi(-\beta)
$$

where the $p_{\mathrm{f} 1}$ is used to present the first-order approximation of the failure probability, $\Phi(\cdot)$ denotes the standard Normal CDF.

The first-order approximation in FORM generally works well because the neighborhood of the design point has dominant contribution to the failure probability. However, if the limit state surface $H(\mathbf{u})=0$ is strongly non-flat or the optimization problem in Equation (12) has multiple local or global solutions, the first-order approximation may not work well. The SORM is driven by improving the capability of the approximation when $H(\mathbf{u})=0$ is non-flat.

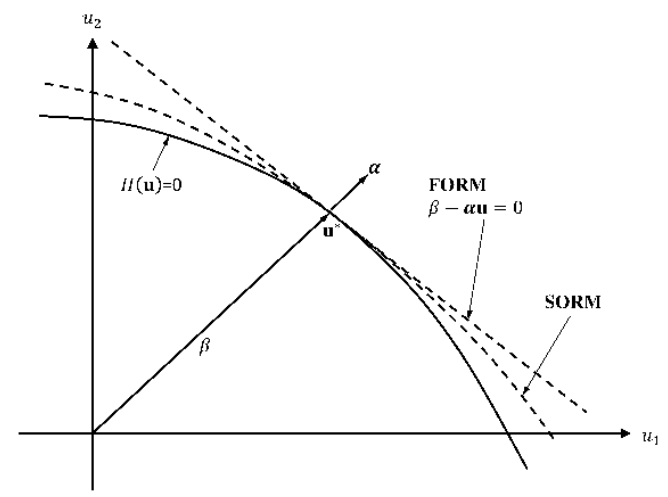

Figure 2. FORM and SORM approximations of limit state function

\subsubsection{Second-Order Reliability Method (SORM)}

The second-order reliability method (SORM), as its name implies, approximates the limit state function $H(\mathbf{u})=0$ by the second-order Taylor expansion [16]0 at design point $\mathbf{u}^{*}$

$$
\begin{aligned}
& H(\mathbf{u}) \cong H_{2}(\mathbf{u})=\nabla H\left(\mathbf{u}^{*}\right)\left(\mathbf{u}-\mathbf{u}^{*}\right)+\frac{1}{2}\left(\mathbf{u}-\mathbf{u}^{*}\right)^{\mathrm{T}} \mathbf{H}\left(\mathbf{u}-\mathbf{u}^{*}\right) \\
& =\left\|\nabla H\left(\mathbf{u}^{*}\right)\right\|\left[(\beta-\alpha \mathbf{u})+\frac{1}{2\left\|\nabla H\left(\mathbf{u}^{*}\right)\right\|}\left(\mathbf{u}-\mathbf{u}^{*}\right)^{\mathrm{T}} \mathbf{H}\left(\mathbf{u}-\mathbf{u}^{*}\right)\right]
\end{aligned}
$$

where $\mathrm{H}_{2}(\mathbf{u})$ denotes the second-order approximation of the limit state function, $\nabla H(\mathbf{u}), \alpha$ and $\beta$ are as defined earlier in Section 3.2.2, and $\mathbf{H}$ is the Hessian matrix at the design point with elements $H_{i j}=\partial^{2} H\left(\mathbf{u}^{*}\right) /\left(\partial U_{i} \partial U_{j}\right)$ for $i, j=1, \cdots, n$.

Since the limit state function is approximated by second-order Taylor expansion, the limit state surface is approximated by a paraboloid in SORM rather than by a linearized plane in FORM. In order to obtain the second-order approximation of the failure probability, the curvatures of the paraboloid need to be evaluated. This is implemented by space rotation and matrix decomposition detailed subsequently.

Based on asymptotic approximations, Bretiung [17] propsed a simple formula for second-order approximation of the failure probability $p_{\mathrm{f} 2}$ calculation, that is,

$$
p_{\mathrm{f}} \cong p_{\mathrm{f} 2}=\Phi(-\beta) \prod_{i=1}^{n-1} \frac{1}{\sqrt{1+\psi(\beta) \kappa_{i}}}
$$


where $\psi(\beta)$ is obtained by $\psi(\beta)=\phi(-\beta) / \Phi(-\beta)$ and for simplify the calculation usually $\psi(\beta)=\beta$ is used, and $\kappa_{i}, i=1, \cdots, n-1$ represents the principal curvatures of the limit state function at the minimum distance point. From the above formula, we can see that each term $1 / \sqrt{1+\psi(\beta) \kappa_{i}}$ acts as a correction factor on the FORM approximation to account for the curvature $\kappa_{i}$ of the limit state surface in the principal direction. Tvedt [18] proposed a three-term approximation in which the last two terms can be interpreted as correctors to Breitung's form. More accurate closed form formulas were derived using Maclaurin series expansion and Taylor series expansion [19][20]. These formulas generally work well in the case of a large curvature radius and a small number of random variables.

In SORM, the Hessian matrix at the design point, $\mathbf{H}$, need to be computed. When performance function is not explicitly known, usually, a finite difference scheme is used to compute the Hessian matrix. The search of design point is required both in FORM and SORM. To solve the optimization problem of design point search, multiple evaluations of performance function are necessary. If the evaluating of performance function is computationally expensive, the cost of the design point search can be prohibitive such that the design point-based methods are not computationally suitable.

\subsection{Response Surface Method (RSM)}

In many practical problems the performance function may not be known explicitly. Instead, it may be known only implicitly, through a procedure such as a finite element analysis, and is expensive to evaluate. This means that, in this case, the safe domain can be defined only through point-by-point 'discovery' such as through repeated numerical analysis with different input values. In these conditions, it is apparent that the FORM and SORM and related methods, which require a closed and preferably differentiable form for the performance function, are not immediately applicable. This has led to the development of Response Surface Method (RSM) for reliability analysis. In RSM, the implicit performance function $G(\mathbf{X})$ is replaced by an artificially constructed function $\hat{G}(\mathbf{X})$, generally a polynomial, around the design point. The typical and commonly used polynomial response surface method is presented here.

Response surface is a collection of mathematical and statistical techniques for exploring the relationships between independent variables and response variables [21]. The polynomial response surface method uses a polynomial function of the input random variables to replace the implicit performance function. The undetermined coefficients in this polynomial are determined so as to minimize the error of approximation, particularly in the region around the design point.

Since the actual limit state function is known only through discrete outcomes, its form and degree are not known, nor can estimate the design point, yet. It means that there is little guidance for the selection of the approximating function $\hat{G}(\mathbf{X})$. However, a second order polynomial is most often employed for the response surface, that is,

$$
\hat{G}(\mathbf{X})=A+\mathbf{X}^{\mathrm{T}} \mathbf{B}+\mathbf{X}^{\mathrm{T}} \mathbf{C X}
$$

where the undetermined (regression) coefficients are denoted by scalar $A$, vector $\mathbf{B}=\left[b_{1}, b_{2}, \cdots, b_{n}\right]^{\mathrm{T}}$, and matrix

$$
\mathbf{C}=\left[\begin{array}{llll}
c_{11} & c_{12} & \cdots & c_{1 n} \\
& c_{22} & \cdots & c_{2 n} \\
& & \ddots & \vdots \\
s y m & & & c_{n n}
\end{array}\right] .
$$

The regression coefficients can be estimated by least squares method with a series of structural analysis with input variables selected according to some Design of Experiments (DOE). In practice, the Central Composite Design (CCD) are usually used to in RSM. In CCD, $2 n+1$ samples are generated at the selected center point $\mathbf{x}_{0}$ and along the line parallel to each coordinate axes at $x_{0 j} \pm f \sigma_{X_{j}}$, where $x_{0 j}$ is the $j$-th component of the center point $\mathbf{x}_{0}, \sigma_{X_{j}}$ is the standard deviation of $X_{j}$, and $f$ is a positive factor, which 
can be chosen be from $1 \sim 3$. With the sampling set from DOE, the response surface function $\hat{G}(\mathbf{X})$ can be constructed. Once $\hat{G}(\mathbf{X})$ has been obtained, the FORM can be used for to calculate the reliability index and the design point.

The goal of response surface method lies in estimating the probability of failure. So that the main interest is in the region of maximum likelihood within the failure domain, i.e., the design point. However, the design point is not known initially. An iterative search technique has been suggested to find the design point. The iterative technique can be described as the following steps:

1. Choose an initial center point $\mathbf{x}_{0}$, generally the mean values point of the random variables $\mu_{x}$ is chosen as the initial point;

2. Built the response approximation $\hat{G}(\mathbf{x})$ : the structural responses are analyzed at the pints from DOE (center point $\mathbf{x}_{0}$ and points given by $x_{0 j} \pm f \sigma_{X_{i}}$ ), and estimate regression coefficients by least squares method in light of the input points and structural responses of DOE.

3. With the approximate performance function $\hat{G}(\mathbf{X})$ obtained in step 2$)$, the reliability index $\beta$ and design point $\mathbf{x}^{*}$ are calculated using FORM.

4. With the design point $\mathbf{x}^{*}$, a new center point $\mathbf{x}_{0}$ can be get by linear interpolation, as:

$$
x_{0}^{i+1}=x_{0}^{i}+\left(G\left(x_{0}^{i}\right) /\left[G\left(x_{0}^{i}\right)-G\left(x^{* i}\right)\right]\right)\left(x^{* i}-x_{0}^{i}\right)
$$

where the superscripts $i$ and $i+1$ refer the $i$-th and $i+1$-th iteration of the procedure, the $G(\cdot)$ is the real performance function, $x_{0}^{i}$ and $x^{* i}$ are the center point and design point of $i$-th iteration, and $x_{0}^{i+1}$ is the center point of $i+1$-th iteration.

5. Repeat step 2) to 4) until convergence is achieved.

The speed with which this iterative technique obtains a sufficiently accurate fit depends on the selection of $f$ and the shape of the actual (but implicit) limit state function in the region being explored. If the iterative scheme does not converge, RSM will fail. Additionally, when the limit state surface cannot be accurately approximated by polynomial, the accuracy of polynomial RSM is not guaranteed.

\section{Illustrative Example}

In this section, a highly nonlinear problem is solved both by FORM and SORM. The performance function is

$$
G(\mathbf{X})=X_{1}^{4}+2 X_{2}^{4}-20
$$

where $X_{1}$ and $X_{2}$ are normal distributed random variables with mean values $\mu_{X_{1}}=\mu_{X_{2}}=10.0$ and standard deviations $\sigma_{X_{1}}=\sigma_{X_{2}}=5.0$. The reliability index and the failure probability are computed by FORM and Bretiung's SORM methods.

\section{FORM Solution}

The basic random variables and the performance function are firstly transformed into standard normal space by

$$
\begin{array}{ll}
U_{i}=T\left(X_{i}\right)=\left(X_{i}-\mu_{X_{i}}\right) / \sigma_{X_{i}} & \text { for } i=1,2 \\
X_{i}=T^{-1}\left(U_{i}\right)=\sigma_{X_{i}} U_{i}+\mu_{X_{i}} \quad \text { for } i=1,2
\end{array}
$$


Then, the performance function in $\mathbf{U}$-space is obtained by

$$
H(\mathbf{U})=G\left(T^{-1}(\mathbf{U})\right)=\left(5 U_{1}+10\right)^{4}+2 \times\left(5 U_{2}+10\right)^{4}-20
$$

The gradient of limit state function $H(\mathbf{U})$ is computed

$$
\nabla H(\mathbf{U})=\left[\frac{\partial H(\mathbf{U})}{\partial U_{1}}, \frac{\partial H(\mathbf{U})}{\partial U_{2}}\right]=\left[20\left(5 U_{1}+10\right)^{3}, 40\left(5 U_{2}+10\right)^{3}\right]
$$

The HL-RF algorithm is used to find the design point $u^{*}$ and compute the reliability index $\beta$. The results of the FORM are

$$
\beta=2.3255, \quad p_{\mathrm{f} 1}=\Phi(-\beta)=0.010024
$$

The design point in $\mathbf{U}$-space is $\mathbf{u}^{*}(-1.6443,-1.6443)^{\mathrm{T}}$ and corresponding point in $\mathbf{X}$-space is $\mathbf{x}^{*}(1.7783,1.7783)^{\mathrm{T}}$.

\section{SORM Solution}

Based on the solution of FORM, the principal curvature of the limit state function is computed as $\kappa_{1}=5.9644$. Thus, the failure probability of SORM is

$$
p_{\mathrm{f} 2} \cong \Phi(-\beta) \frac{1}{\sqrt{1+\frac{\phi(-\beta)}{\Phi(-\beta)} \kappa_{1}}}=0.002438
$$

From above FORM and SORM results, $p_{\mathrm{f} 1}=0.010024$ and $p_{\mathrm{f} 2}=0.002438$, we can find that the failure probabilities computed by FORM and SORM are significantly different for this highly nonlinear problem. Thus, for problems with nonlinear limit state, the accuracy of FORM cannot be assured.

\section{Summary}

In this paper, local reliability methods, which use local approximation of the limit state function (or performance function) in failure probability calculation, have been presented. From above representations and others' research works, it could be concluded that the main drawback of local reliability methods is that they do not give exact results for most practical reliability problems of structures, especially the problems with highly nonlinear performance function.

The simplest MVFOSM method only provides reasonably accurate results when the performance function is nearly linear and the input random variables are approximately Normal distributed. This limitation somewhat has been overcome by the design point-based methods. For all the design pointbased methods, the search of the design point is an essential task. For this reason, the fails in solving the optimization problem of design point search will consequently lead to fails for this kind of methods. From this point, for these reliability problems in which the convergence of design point searching cannot be obtained, the design point-based methods cannot work. Moreover, for nonlinear limit state functions, the FORM cannot provide accurate failure probability due to its low-order approximation of the limit state function. The SORM and RSM can somewhat overcome this drawback and improve the reliability estimation for nonlinear problems by approximating the limit state with high order functions (secondorder expansion and second order polynomial). However, these two methods, SORM and RSM, may not guarantee the accuracy of the results when the limit state is highly nonlinear and possess multiple local solutions to the design point search problem. In addition, when the performance functions are expensive to evaluate, the FORM and SORM will be prohibitive due to the computational cost in design point search. The polynomial response surface method, of course, has its limitations. As previously stated, the RSM may abort due to the convergence in tis iterative algorithm. In situations when the limit state surface cannot be accurately approximated by polynomial, the accuracy of polynomial RSM is not guaranteed. 


\section{References}

[1] El Hami A., \& Bouchaib R. Uncertainty and Optimization in Structural Mechanics. John Wiley \& Sons, 2013.

[2] Bastidas-Arteaga E., \& Soubra A.H., Reliability Analysis Methods. ALERT Doctoral School 2014-Stochastic Analysis and Inverse Modelling, pp. 53-77, 2014.

[3] Lemaire M., Structural reliability. John Wiley \& Sons, 2013.

[4] Bichon B.J., Eldred M.S., Swiler L.P., Mahadevan S., \& McFarland J.M., Multimodal reliability assessment for complex engineering applications using efficient global optimization. AIAA Paper No. AIAA-2007-1946, 2007.

[5] El Hami A., \& Radi B., Incertitudes, optimisation et fiabilité des structures. Hermès science publications, 2013.

[6] Choi S.K., Grandhi R., \& Canfield R.A., Reliability-based structural design. Springer Science \& Business Media, 2006.

[7] Ditlevsen O., \& Madsen H.O., Structural reliability methods (Vol. 178). New York: Wiley, 1996.

[8] Hurtado J.E., Structural reliability: statistical learning perspectives. Springer Science \& Business Media, 2013.

[9] Nikolaidis E., Ghiocel D.M., \& Singhal S. (Eds.). Engineering design reliability handbook. CRC Press, 2004.

[10] Liu P.L., \& Der Kiureghian A., Multivariate distribution models with prescribed marginals and covariances. Probabilistic Engineering Mechanics, vol. 1, no. 2, pp.105-112, 1986.

[11] Bucher C., Methods of Reliability Analysis in the context of RDO. Proceedings Weimarer Optimierungs-und Stochastiktage WOST, 8, 2011.

[12] Cornell C.A., A probability-based structural code. ACI Journal, no. 66, pp. 974-985, 1969.

[13] Thoft-Cristensen P., \& Baker M.J., Structural reliability theory and its applications. Springer Science \& Business Media, 2012.

[14] Hasofer A.M., \& Lind N.C., Exact and invariant second-moment code format. Journal of the Engineering Mechanics division, vol. 100, no. 1, pp. 111-121, 1974.

[15] Rackwitz R., \& Flessler B., Structural reliability under combined random load sequences. Computers \& Structures, vol. 9, no. 5, pp. 489-494, 1978.

[16] Der Kiureghian A., Lin H.Z., \& Hwang S.J., Second-order reliability approximations. Journal of Engineering Mechanics, vol. 113, no. 8, pp. 1208-1225, 1987.

[17] Breitung K., Asymptotic approximations for multinormal integrals. Journal of Engineering Mechanics, vol. 110, no. 3, pp. 357-366, 1984.

[18] Tvedt L., Second order reliability by an exact integral. In Reliability and Optimization of Structural Systems' 88 (pp. 377-384). Springer Berlin Heidelberg, 1989.

[19] Köylüoğlu H.U., \& Nielsen S.R., New approximations for SORM integrals. Structural Safety, vol. 13, no. 4, pp. 235-246, 1994.

[20] Cai G.Q., \& Elishakoff I., Refined second-order reliability analysis. Structural Safety, vol. 14, no. 4, pp. 267-276, 1994.

[21] Myers R.H., Montgomery D.C., \& Anderson-Cook C.M., Response surface methodology: process and product optimization using designed experiments. John Wiley \& Sons, 2016. 